\title{
Gravity waves generated by convection in the Darwin area during the Darwin Area Wave Experiment
}

\author{
M. Joan Alexander \\ Colorado Research Associates, Division of Northwest Research Associates, Boulder, Colorado, USA
}

Peter T. May

Bureau of Meteorology Research Center, Melbourne, Victoria, Australia

Jadwiga H. Beres

National Center for Atmospheric Research, Boulder, Colorado, USA

Received 2 March 2004; revised 4 June 2004; accepted 29 June 2004; published 10 September 2004.

[1] We describe a model study of gravity wave generation by convection near Darwin during the Darwin Area Wave Experiment (DAWEX). We focus on an 7-hour period on 17 November 2001 for our study which included a major Hector event over the Tiwi Islands followed by a continental convective outbreak that included a squall line to the southeast of Darwin. Our model is a dry version of a three-dimensional (3-D) mesoscale cloud resolving model with horizontally uniform background wind and stability fields. The model is forced with a spatially and temporally varying heating field representative of the convective latent heating in the area. We derive this heating field from 3-D volumetric reflectivity from the precipitation radar located just north of Darwin at Gunn Point during DAWEX. The conversion from radar reflectivity to a 3-D latent heating field requires numerous assumptions that do not allow a quantitative heating estimate but which do provide a very realistic measure of the spatial morphology and temporal variations of the latent heating. Gravity waves generated by convective heating are known to be very sensitive to these characteristics of the heating, so our resulting wave field is likely to be the most realistic possible description of the waves emitted from convection in the Darwin area. Uncertainty in the wave amplitudes is large both because the input forcing (heating) is uncertain and because the use of heating as the sole proxy for wave forcing leads to additional wave amplitude errors. We therefore compare the results of the model to other DAWEX measurements and previous modeling studies both to validate the waves in our model and to calibrate the wave amplitudes. Our study in turn aids in the interpretation of the other gravity wave measurements during DAWEX. INDEX TERMS: 3314 Meteorology and Atmospheric Dynamics: Convective processes; 3334 Meteorology and Atmospheric Dynamics: Middle atmosphere dynamics (0341, 0342); 3337 Meteorology and Atmospheric Dynamics: Numerical modeling and data assimilation; 3362 Meteorology and Atmospheric Dynamics: Stratosphere/troposphere interactions 3384 Meteorology and Atmospheric Dynamics: Waves and tides; KEYWORDS: gravity wave, convection, latent heating, radar

Citation: Alexander, M. J., P. T. May, and J. H. Beres (2004), Gravity waves generated by convection in the Darwin area during the Darwin Area Wave Experiment, J. Geophys. Res., 109, D20S04, doi:10.1029/2004JD004729.

\section{Introduction}

[2] The Darwin Area Waves Experiment (DAWEX) was designed to observe gravity waves generated by convection at levels in the atmosphere ranging from the surface to the upper atmosphere. The DAWEX observations are described in the work of K. Hamilton et al. (Darwin Area Wave Experiment field campaign to study gravity wave genera-

Copyright 2004 by the American Geophysical Union. 0148-0227/04/2004JD004729\$09.00 tion and propagation, submitted to Journal of Geophysical Research, 2004, hereinafter referred to as Hamilton et al., submitted manuscript, 2004). They include boundary layer radar observations between the surface and $8 \mathrm{~km}$, radiosonde balloon profiles from the surface to $\sim 30 \mathrm{~km}$, airglow imaging measurements from the mesopause region, and more. Inclusion of smaller-scale gravity wave effects on the large-scale atmospheric circulation is currently a poorly constrained parameterized process in global models. These effects depend on the spectral characteristics of the waves, which in turn depend on the details of unresolved wave 
sources. Providing constraints for parameterizations of global effects of convectively generated gravity waves was the motivation for DAWEX.

[3] This paper describes a unique model study that generates a realistic representation of both the time history and geographic variations in gravity waves generated by convection in the Darwin, Australia area during DAWEX. We focus on a 7-hour period of active convection in the region on 17 November 2001. This period includes both a major Hector event followed by a continental squall line. (See Hamilton et al. (submitted manuscript, 2004) for a description of Hector and other DAWEX convection.) The 17th of November was also chosen to accommodate data coverage issues with the other DAWEX measurements for comparison to our model results.

[4] Our method first uses three-dimensional (3-D) precipitation radar measurements of reflectivity to estimate realistic spatial and temporal patterns in the latent heating within the convection in a $128-\mathrm{km}$ radius field surrounding the radar site near Darwin. We then use these spatially and temporally varying heating distributions to force a field of gravity waves in a numerical mesoscale model. The resulting 3-D, time-dependent model fields are compared to the boundary layer radar and radiosonde measurements, and used to characterize the propagation properties of the vertically propagating gravity waves in the stratosphere.

[5] Several previous 3-D model studies of gravity waves generated by convection have used cloud-resolving models with parameterized precipitation microphysics [Piani et al., 2000; Piani and Durran, 2001; Lane and Reeder, 2001; Horinouchi et al., 2002]. Although cloud-resolving models of moist convection continue to improve in their accuracy for describing and predicting observed storms [e.g., Crook, 2001; Xue et al., 2003], they cannot generally develop the correct timing, location, and duration of storm events, particularly when such details of the individual rain shafts are needed, as for the present problem of gravity wave generation for DAWEX. Such details of the precipitation are simply too stochastic for this approach. However, observations of precipitation from radar do capture these details, and these details are now known to be important for determining the characteristics of the gravity waves that are generated.

[6] The use of diabatic or latent heating as a proxy for wave forcing in convection has a long history, beginning with studies of tropical planetary-scale waves [Holton, 1972; Salby and Garcia, 1987; Garcia and Salby, 1987; Manzini and Hamilton, 1993; Bergman and Salby, 1994; Ricciardulli and Garcia, 2000]. Several of these studies used satellite infrared cloud imagery as a proxy for determining the horizontal, vertical and temporal scales of variation of latent heating in deep convection. Others used properties of parameterized convection in global models to estimate these properties of the heating. This restricted these studies to larger horizontal-scale and longer timescale variability and waves. Our approach of using radar observations gives a clear picture of the small horizontal-scale and short timescale latent heating structure within clouds that is known to be important in determining the properties of the gravity waves that are generated.

[7] Salby and Garcia [1987] found a relationship between the depth scale of the heating and the vertical wavelengths of the waves generated with only relatively minor sensitivity to the profile shape. More recently, studies of smaller-scale gravity wave generation in mesoscale simulations of moist convection have found similar relationships between the depth scale of the heating and the vertical wavelengths of waves generated [Alexander et al., 1995; Pandya and Alexander, 1999; Piani et al., 2000; Beres et al., 2002]. However, for gravity waves, the depth scale of the heating alone does not predict the characteristics of the waves. Both the horizontal and vertical scales as well as the time history of the heating are now known to be important predictors of the characteristics of the waves [Holton et al., 2002; Beres et al., 2004; Alexander and Holton, 2004]. Shear in the horizontal winds in the vicinity of the heating is another important factor determining the wave characteristics [Beres et al., 2002; Chun and Baik, 2002].

[8] In addition to latent heating within a storm there are heat and momentum flux convergences that can also force small-scale waves [Lane et al., 2001]. Song et al. [2003] evaluated these terms in a 2-D cloud-resolving model, then forced a dry version of the same model with the different components of the forcing and examined the resulting gravity wave characteristics. Their study found that each of the forcing terms in the thermodynamic and momentum equations generated very similar spectral characteristics of gravity waves, however, the individual terms tended to over-estimate the wave amplitudes, in agreement with previous studies comparing waves generated in full physics models with those generated in linear models by latent heating alone [Manzini and Hamilton, 1993; Pandya and Alexander, 1999]. The different thermodynamic and momentum forcing terms have similar morphologies in space and time, but destructively interfere giving partial cancellation of the net forcing and weaker overall wave amplitudes [Song et al., 2003].

[9] In this study we convert radar reflectivity to latent heating distributions, which are then used to force waves in a 3-D dry mesoscale model. This approach provides the best available representation of the geographical and temporal patterns in the gravity wave forcing. Although methods for precipitation retrieval from radar observations continue to improve in their accuracy [Steiner et al., 2004], our heating distributions will include large uncertainties in the shape of the vertical profile of the heating and the overall magnitudes of the heating input to the model. Further, the wave amplitudes generated by heating alone will be different than those that would be forced if we could somehow include the additional momentum and heat flux convergence forcing terms [Song et al., 2003]. Observations cannot hope to provide all these details of the forcing, but the studies cited above have shown that the spatial and temporal variations in the wave forcing are key to capturing the essential spectral characteristics of the waves generated.

[10] The above studies collectively suggest that our use of the radar reflectivity to describe the wave forcing will give the most realistic possible time history and geographic variability in the wave field generated by convection during DAWEX, but that the wave field in this model will require some kind of calibration of the wave amplitudes. For this purpose we also discuss comparisons between the model wave field and DAWEX observations. 

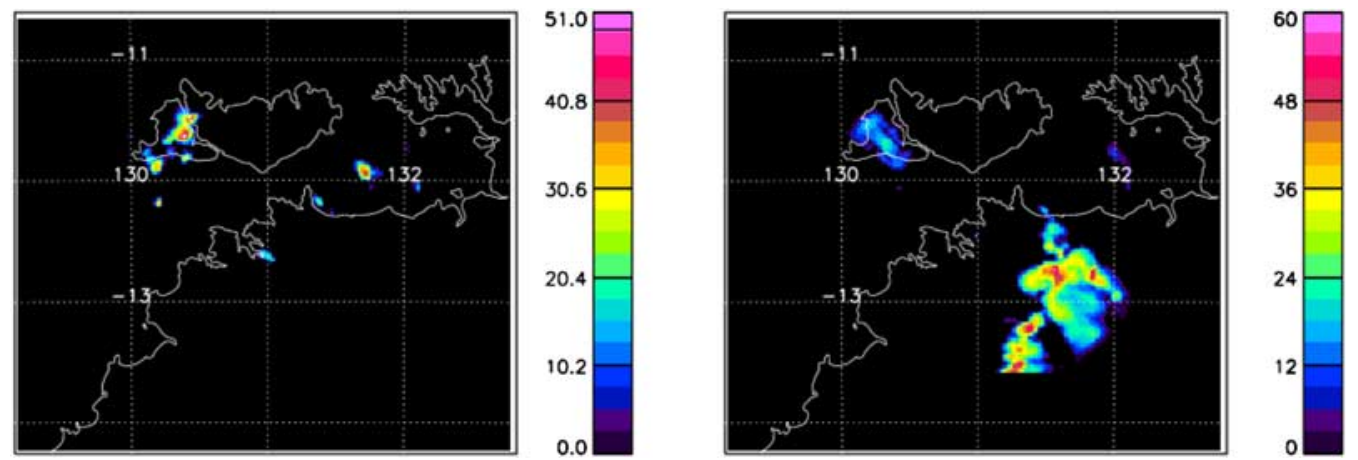

Figure 1. Radar reflectivity at $8 \mathrm{~km}$ altitude mapped in decibels (dBZ) for two times on 17 November: (left) 1330 LT (0400 UT), (right) 1730 LT (0800 UT).

[11] Parameterizations of small-scale waves generated by convection are still under development [Beres et al., 2004], but current approaches are based on statistical properties of unresolved latent heating. Our study provides a point of comparison for constraining these parameterizations, and comparison of our results with such parameterizations is the subject of our ongoing work.

\section{Data Analysis}

[12] Our study focuses on a 7-hour period on 17 November 2001, from 0300-0950 UT (1230-1920 LT). Here we describe the radar data and the conversion from radar reflectivity to heating rates.

\subsection{C-Pol Radar Data}

[13] The C-Pol radar is described in the work of Keenan et al. [1998] and in the overview paper by Hamilton et al. (submitted manuscript, 2004), which includes a description of DAWEX weather conditions. For this study, the data was processed to provide volumetric reflectivity at $2-\mathrm{km}$ horizontal resolution in a $128-\mathrm{km}$ radius area at $1-\mathrm{km}$ vertical resolution every $10 \mathrm{~min}$. Two examples of radar reflectivity at selected times are shown in Figure 1. Figure 1a shows an example of reflectivity when a major Hector storm appears over the Tiwi Islands, and $1 \mathrm{~b}$ shows a later time when a squall line appears over the continental region. The reflectivity measurements are sensitive to precipitation with the highest sensitivity to the largest precipitation particles. The spatial and temporal patterns in reflectivity should provide the best available estimates of the spatial and temporal patterns in precipitation and latent heating during DAWEX.

\subsection{Estimate of Latent Heating}

[14] At the detail level, the conversion of radar reflectivity to precipitation cannot be made directly without recognition of the uncertainties and errors in the conversion. For example, precipitation particle growth is fast compared to the advection time scale for lofting these particles in strong updrafts. The vertical profile of reflectivity is therefore a poor representation of the vertical profile of heating. The precipitation also falls, particularly the largest particles, those to which the radar is most sensitive. In addition, ice has a smaller refractive index than liquid water, so that ice crystals of a given mass will have smaller radar cross sections than equivalently sized liquid drops. The melting level is thus known to give a "bright band" of reflectivity at the $5-\mathrm{km}$ level in the stratiform cloud layers in these data, but the effect is weak or absent in the convective portion of clouds. Thus the radar reflectivity at the melting level does not provide a good proportional measure of latent heat release in the cloud.

[15] We know from theoretical studies that the vertical wavelengths and phase speeds of waves generated by a heat source are dependent on the vertical structure of the heating [e.g., Salby and Garcia, 1987; Pandya et al., 1993; Alexander et al., 1995; Holton et al., 2002; Alexander and Holton, 2004]. Some of these studies suggest there is no one-to-one correspondence between the Fourier spectrum of the heating profile and the Fourier spectrum of the waves generated, because other factors including the horizontal
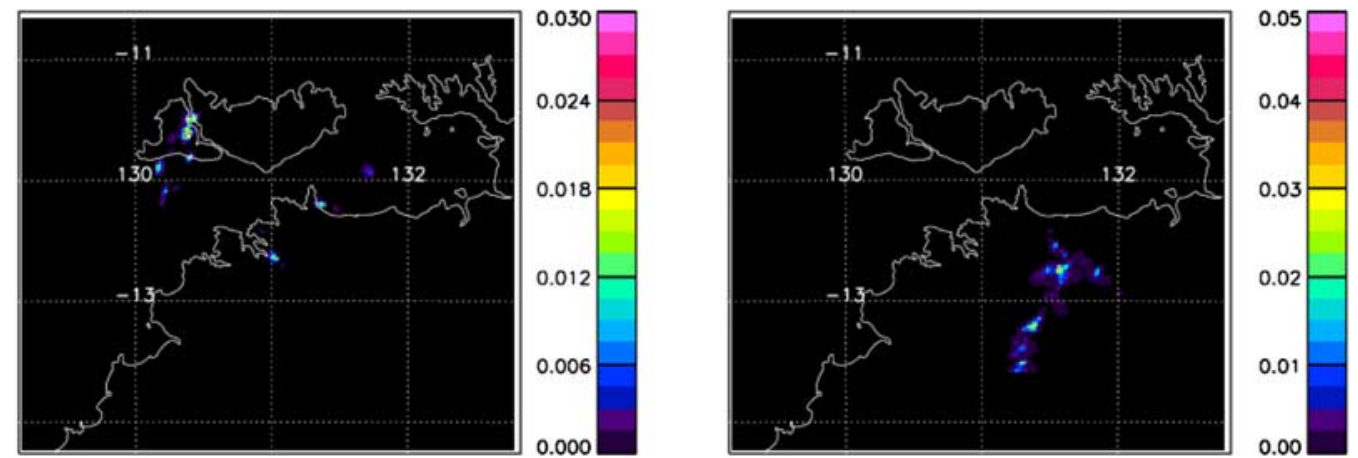

Figure 2. Column integrated heating $(\mathrm{K} / \mathrm{s})$ for the same two times shown in Figure 1: (left) 1330 LT (0400 UT), (right) 1730 LT (0800 UT). 

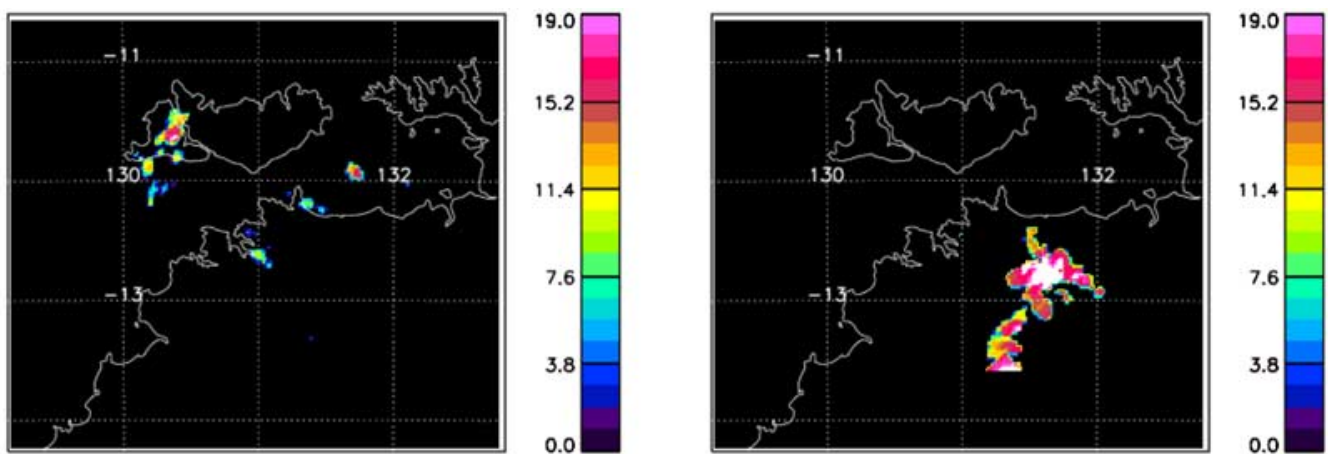

Figure 3. Maximum radar echo heights $z_{\text {top }}(\mathrm{km})$ for column heating $>0.1 \%$ of maximum values. Here $z_{\text {top }}$ is the altitude where the heating profile goes to zero: (left) 1330 LT (0400 UT), (right) 1730 LT (0800 UT).

scale and time-dependence of the heating combine to determine the vertical wavelengths of the waves generated.

[16] Because of these uncertainties in the conversion of a reflectivity profile to a heating profile, we choose a simple profile shape and parameterize the depth and magnitude of the heating profile based on the radar reflectivity measurements. We first compute the vertically integrated column reflectivity at each horizontal grid point, omitting the $5-\mathrm{km}$ melting layer to prevent disproportionately emphasizing the stratiform over the convective clouds. We next make the assumption that this column integrated reflectivity is proportional to the column integrated heating. We then redistribute this column heating in a half-sine-wave vertical profile between $950 \mathrm{mb}\left(z_{0}\right)$ and the highest altitude at which significant radar echoes are measured $\left(z_{\text {top }}\right)$, which varies in $1-\mathrm{km}$ steps up to $19 \mathrm{~km}$. We choose $6 \mathrm{dBZ}$ as the threshold for significant radar echo power. (For reference, our largest heating values are associated with small areas of high reflectivity $>50 \mathrm{dBZ}$, which is more than 4 orders of magnitude larger than our $6 \mathrm{dBZ}$ threshold.) If no reflectivity values exceed $6 \mathrm{dBZ}$ in the column, then the column heating at that point is set to zero. We have also tapered the heating rates at the edge of the circular radar field with a $5-\mathrm{km}$ wide taper function to prevent sharp discontinuities at the edge of the radar-derived heating field. Without the taper, these might produce spurious short horizontal-scale waves in an unrealistic manner.

[17] Two examples of the column-integrated heating are shown in Figure 2. Figures $2 \mathrm{a}$ and $2 \mathrm{~b}$ represent the same times as the reflectivity dBZ maps shown in Figures 1a and $1 \mathrm{~b}$, respectively. Note that because $\mathrm{dBZ}$ units are logarithmic ( $\mathrm{dBZ}=10 \log _{10} R$, where $R$ is the reflectivity), and our heating is proportional to $R$, the maps in Figure 2 show the small area of the major heating events, which are associated with isolated, deep, and intense rainshafts. Peak values of the heating field we input to the model are $\sim 0.04-.05 \mathrm{~K} \mathrm{~s}^{-1}$. These peak values have been purposefully kept at modest values to avoid large nonlinear responses in the model that might be unrealistic and might violate the assumptions inherent in our description of the gravity wave forcing. Figure 3 shows corresponding maps of $z_{\text {top }}$.

\subsection{Time History of the 17 November Case Study}

[18] The 7-hour period we will focus on in the model study begins at 1230 LT (0300 UT) and ends at 1820 LT (0950 UT). The time history of the volume sum of latent heating within the radar area is shown in Figure 4. The period begins quietly with little precipitation in the Darwin area. By 1400 LT, a major Hector event occurs over the Tiwi Islands with peak heating rates of $0.05 \mathrm{~K} \mathrm{~s}^{-1}$. A second peak in the heating rates, occurring near $1800 \mathrm{LT}$, is associated with a squall line propagating into the area. The heating in the first half of our 7-hour period is dominated by the Hector event, and in the second half, by the continental squall line. Peak local values of the heating are similar in both these events.

[19] Figure 5 shows the results of a compact (Debauchies) wavelet analysis that quantifies the scale of the heating events averaged over the first and second halves of the 7 -hour period. The wavelet analysis resolves scales of 4,8 , $16,32,64,128$, and $256 \mathrm{~km}$. Only scales $4-128 \mathrm{~km}$ are shown. During the Hector period, the greatest power (color scale in normalized units) in the heating field occurs at the $8-\mathrm{km}$ scale. There is a virtual absence of power at a scale of $128 \mathrm{~km}$ and very little at scales larger than $32 \mathrm{~km}$. No obvious anisotropy in the zonal and meriodional scales is apparent at the resolution of the wavelet analysis in the first period. The small-scale, isolated heating regions tend to be roughly circular on average, illustrated by the tendency for the maxima in the heating power to occur at points along the diagonal of the plot. During the continental convection period, the scales of the heating are a bit larger, peaking

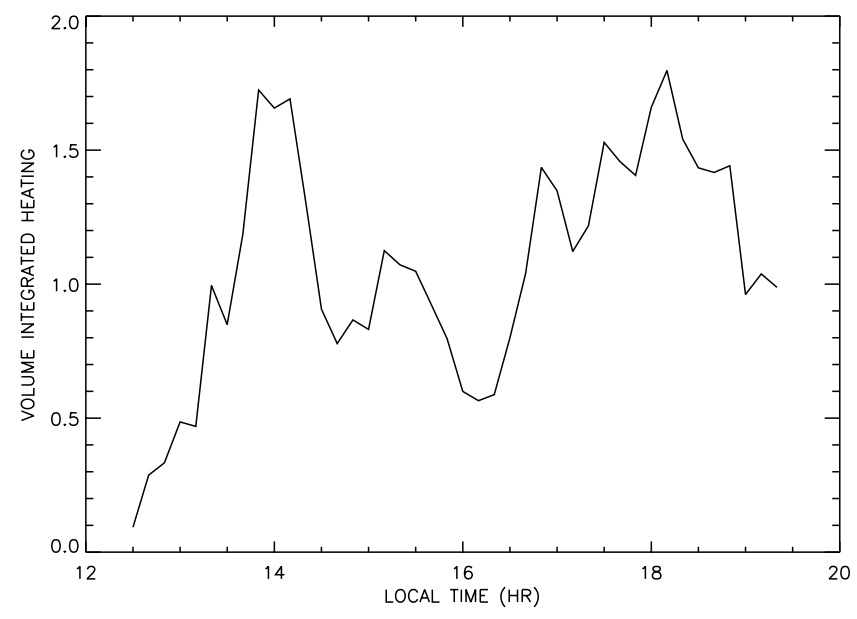

Figure 4. Time series of volume-integrated heating (normalized units) for the period 1330-1730 LT. 

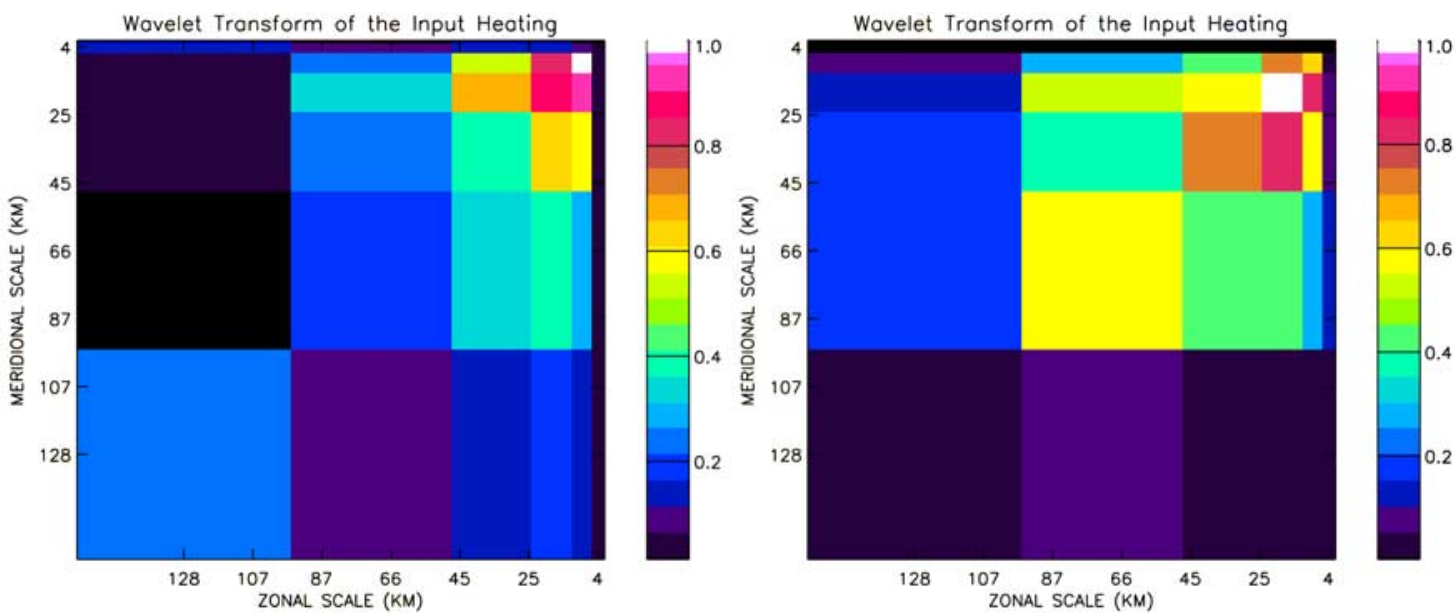

Figure 5. Area averaged power in the heating field (normalized units) as a function of zonal and meridional scale derived from a compact wavelet analysis of the column integrated heating fields. (left) Average over 1230-1520 LT when Hector dominates the heating field. (right) Average over 15301820 LT when continental convection dominates.

now at $16 \mathrm{~km}$, but still very small scale compared to the size of the radar domain. The squall line occurring in this period creates a line of heating structures that is oriented NE to SW. Some tendency for longer meridional scales relative to zonal scales is indicated in this second period reflecting a slight north-south tilt to the squall line orientation.

\section{Numerical Gravity Wave Model Experiment}

\subsection{Model Description}

[20] We performed experiments with a cloud-resolving numerical model [Durran and Klemp, 1983; Piani et al., 2000] with zero moisture. Instead of deriving latent heating from the microphysical parameterization, we input the

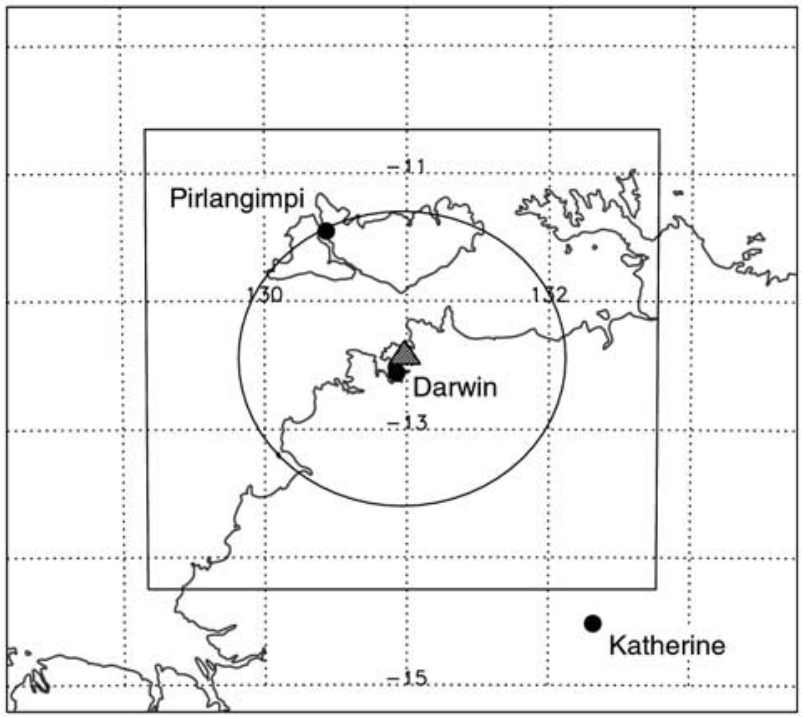

Figure 6. The model domain (solid box outline) is centered on the $\mathrm{C}$-pol radar site (triangle). The input heating field is confined within the $128-\mathrm{km}$ radius circle surrounding the radar. The Tiwi Islands are the island pair centered in the top portion of the radar field. Circles show the sites of 3-hourly radiosonde launches. precomputed heating field derived from the radar reflectivity to force the model. The spatially and temporally varying heating input into the model generates a complex field of gravity waves. The model includes no topography nor any horizontal variations in the background state of any kind, so the only forcing mechanism for perturbations from the mean state is the input heating. Although the input heating stops after 7 hours, we run the model for 8 hours to allow for wave propagation time into the stratosphere.

[21] The model domain is shown in Figure 6 with a superimposed map and a triangle symbol showing the location of the C-pol radar. The box delineates the model domain which is $400 \times 400 \mathrm{~km}$ with $2-\mathrm{km}$ horizontal resolution throughout. The domain depth is $25 \mathrm{~km}$ with $0.25-\mathrm{km}$ vertical resolution. Our model settings are otherwise identical to those in the work of Beres et al. [2004]. As in the work of Beres et al. [2004], the model is run with wavepermeable boundary conditions [Durran et al., 1993] on all
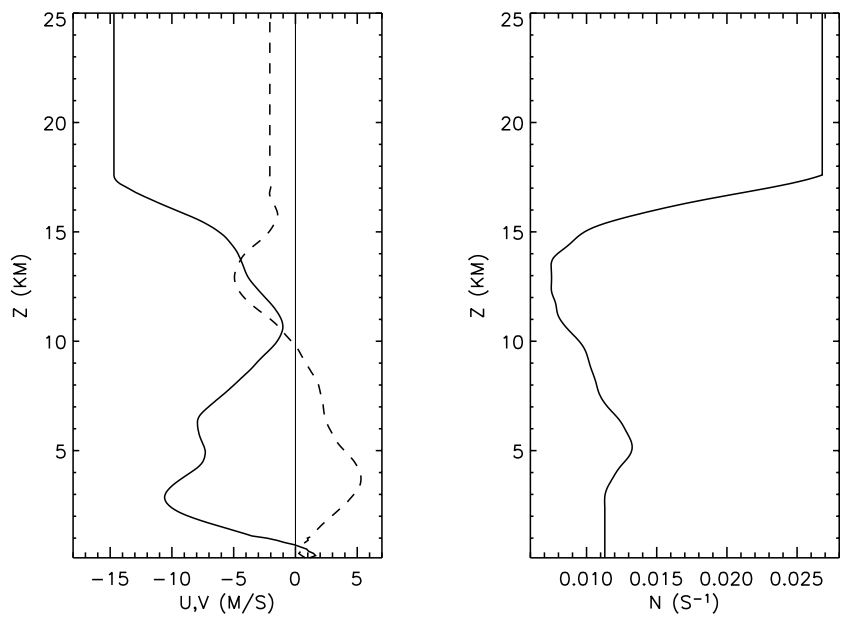

Figure 7. (left) Horizontal wind profiles in $\mathrm{m} \mathrm{s}^{-1}$ and (right) buoyancy frequency in $\mathrm{s}-1$ input as background fields to the model. In the left panel the dashed line shows the meridional wind and solid zonal wind. 

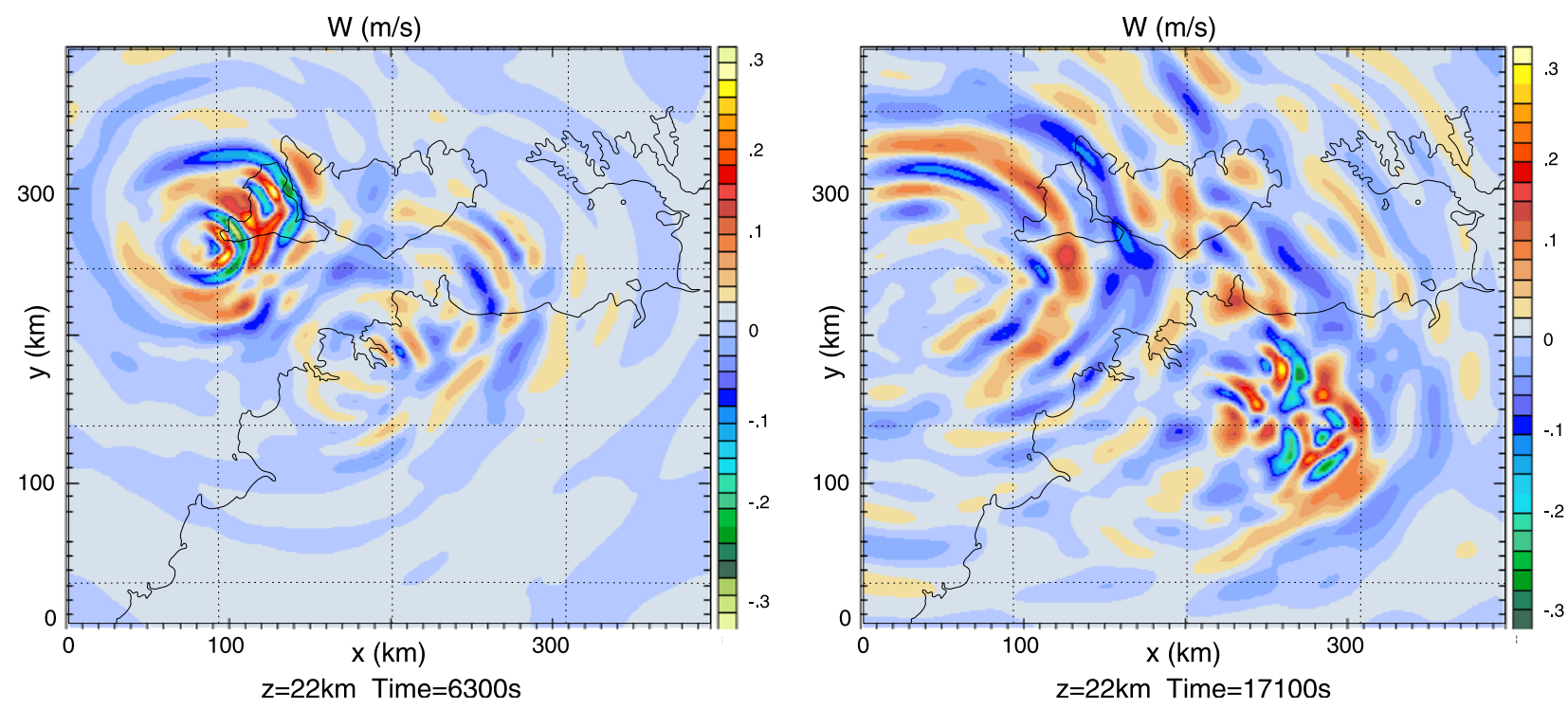

Figure 8. Instantaneous vertical velocity at $22 \mathrm{~km}$ altitude from the model. The times shown are $45 \mathrm{~min}$ after the two input heating fields shown in Figure 2 and represent local times 1415 and 1715.

four sides and at the model top. Figure 6 also shows the circular field into which the radar-derived heating is input. This is a $128-\mathrm{km}$ radius circle centered in the domain.

[22] The background state of the model includes vertical wind and stability variations, which are known to have important effects on the generation and propagation of the gravity waves. These are based on 5-day means of November radiosonde profiles from Garden Point (T. Tsuda et al., Characteristics of atmospheric waves revealed by coordinated radiosonde campaigns during the Darwin Area Wave Experiment, submitted to Journal of Geophysical Research, 2004, hereinafter referred to as Tsuda et al., submitted manuscript, 2004), and are shown in Figure 7. We choose 5-day means to eliminate short horizontal-scale and timescale waves in the profiles, while preserving the important vertical variations in the background state. Individual profiles, shown in the work of Tsuda et al. (submitted manuscript, 2004), show little diurnal variation above the boundary layer, but a gradual progression in wind strength through this period. Our background profiles are set to constant values above $17 \mathrm{~km}$, the altitude of the tropopause. Because the heating profiles sometimes extend up to $19 \mathrm{~km}$, and because shear near the top of the heating profile can be important for the obstacle-effect type of wave generation in convection [Beres et al., 2002], we looked carefully at shear up to $19 \mathrm{~km}$. The shear was very small (almost zero) between 17 and $19 \mathrm{~km}$, so our approximate profiles should generate a realistic spectrum of waves. The 5-day mean profiles at Darwin were very similar to those in Figure 7, and the November profiles are also very similar to the October profiles (see Tsuda et al. (submitted manuscript, 2004))

[23] There is additional shear in the stratosphere above $19 \mathrm{~km}$ associated with a descending westerly phase of the quasibiennial oscillation (QBO) that we have not included in the model background state. These winds will not affect
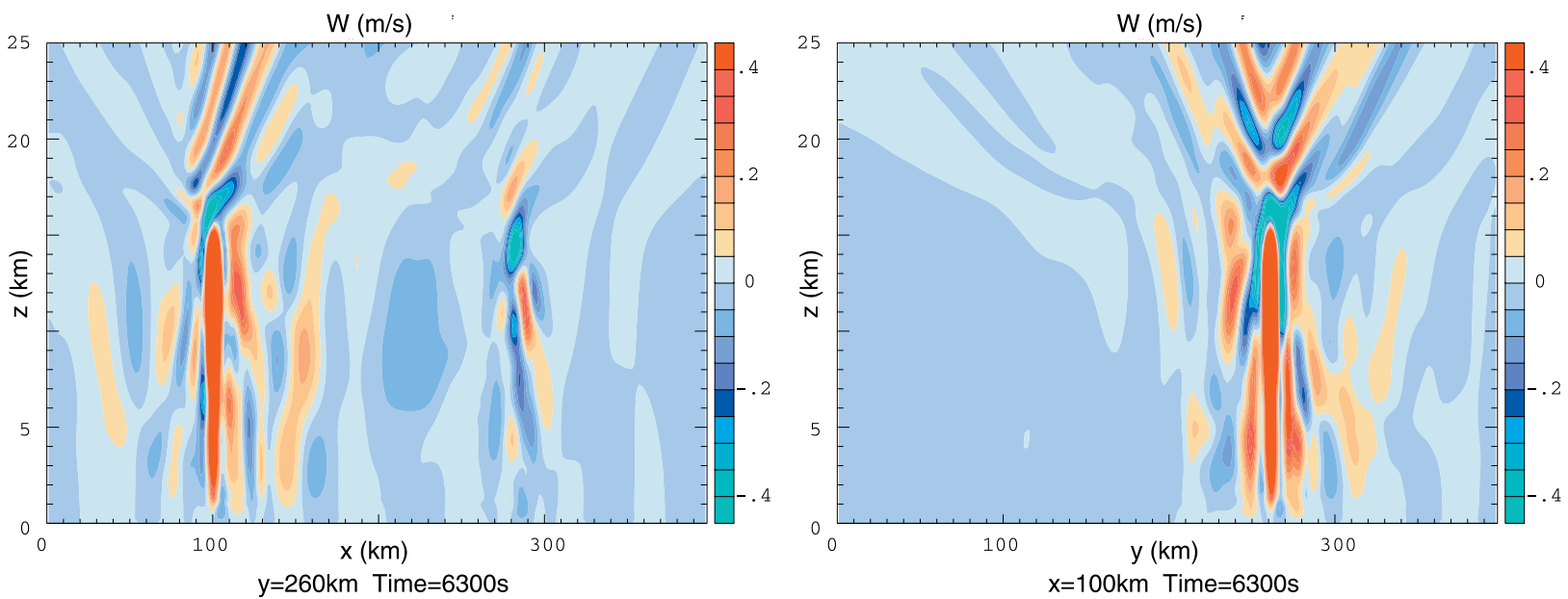

Figure 9. Instantaneous vertical cross sections of $w$ representing 1415 LT. Left panel is an $x-z$ cross section at $y=260 \mathrm{~km}$, and the right panel is an $y-z$ cross section at $x=100 \mathrm{~km}$. The point $(x, y)=$ $(100,260) \mathrm{km}$ is at the center of the strongest waves at this time shown in Figure 8. 

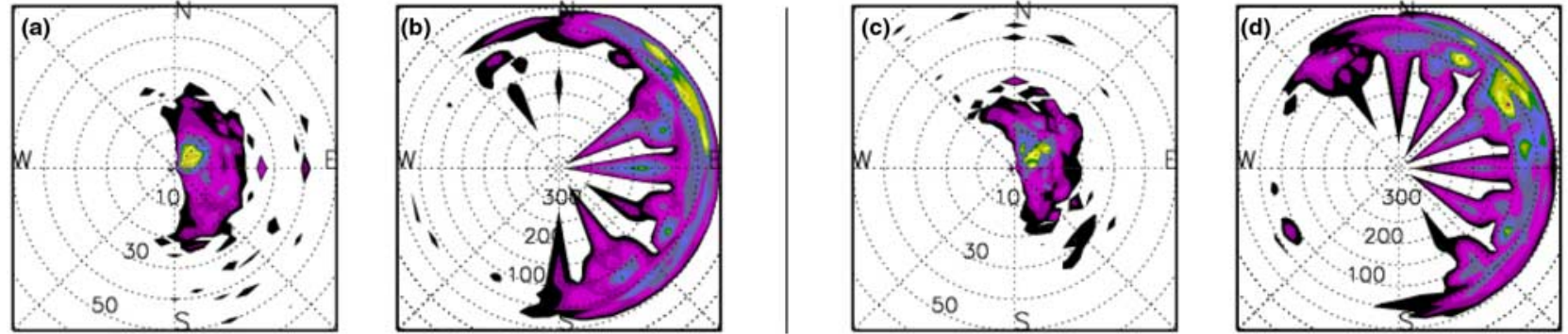

Figure 10. Polar plots of momentum flux spectra versus propagation direction for waves in the stratosphere 21-24.75 km, computed from a 3-D spectral analysis. (a and b) computed from the first 4 hours of simulation time and (c and d) from the second 4 hours. In Figures 10a and 10c the radial axis is phase speed $\left(\mathrm{m} \mathrm{s}^{-1}\right)$, and in Figures $10 \mathrm{~b}$ and $10 \mathrm{~d}$ it is horizontal wavelength $(\mathrm{km})$. The color contours are equally spaced on a linear scale representing percent of the maximum value (see text for further details). Absolute values will be discussed in section 4.

the wave generation, but will affect the transmission of waves into the upper atmosphere. This will be important in the comparison of these results to stratospheric waves seen in the radiosonde profiles as well as to waves observed at the mesopause by the airglow imagers. The upper level winds and their effects on middle atmosphere waves will be discussed further in section 4.

\subsection{Model Results}

[24] The input heating field is variable enough that the resulting wave field in the model displays a wide range of wave properties that vary in both space and time. Two instantaneous vertical velocity $(w)$ fields at $22-\mathrm{km}$ altitude in the stratosphere are shown in Figure 8. These are shown at times 45 min after the input heating fields shown in Figure 2 to allow some propagation time from the heat sources in the troposphere to this level in the stratosphere. Maximum $w$ this figure range $\pm 0.35 \mathrm{~m} \mathrm{~s}^{-1}$. These are fairly small values for gravity waves above deep convection, but remember we have purposefully scaled the estimated heating magnitudes to modest values to keep the model in a linear range, so the wave amplitudes should be considered correspondingly scalable. We will address the issue of calibrating these model results with supplemental observations in section 4 .

[25] Figure 9 shows two vertical cross sections through the center of the large amplitude wave perturbations located near $(x, y)=(100,260) \mathrm{km}$ in Figure 8a. In Figure 9, the vertical velocity color scale has been saturated at $0.4 \mathrm{~m} \mathrm{~s}^{-1}$ in order to show motions in the full altitude range. The strong tropospheric updraft in these figures is just over $11 \mathrm{~m} \mathrm{~s}^{-1}$, which is a common maximum updraft magnitude that occurs in other locations as well during the simulation. Note that an updraft strength of $11 \mathrm{~m} \mathrm{~s}^{-1}$ is a common value seen in the mid troposphere $(\sim 5 \mathrm{~km})$ in comparatively modest convection in the Darwin area [e.g., May et al., 2002], whereas in the type of convection we are modeling here, we expect magnitudes substantially larger than this at
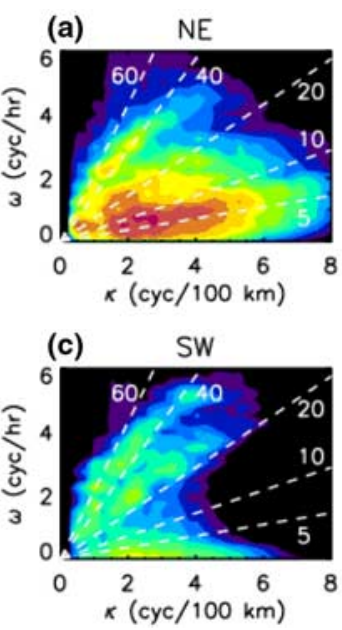
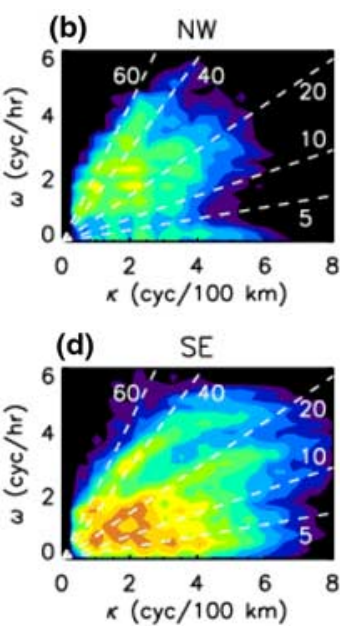
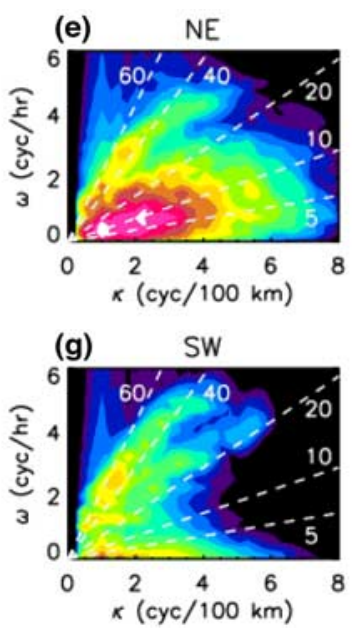
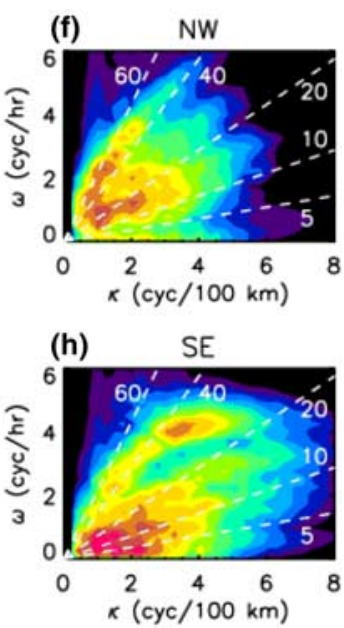

Figure 11. Plots of momentum flux spectra versus horizontal wave number $\kappa$ and frequency $\omega$ for each of four quadrants: northeast (NE), northwest (NW), southwest (SW), and southeast (SE). These are computed from horizontal and vertical wind covariances in the stratosphere, and averaged over altitudes 21-24.75 km as in Figure 10. $(\mathrm{a}-\mathrm{d})$ Results from the first 4 hours of the simulation; $(\mathrm{e}-\mathrm{h})$ for the second 4 hours. Spectra are plotted here with color contours on a log scale, three contours per decade, to better show weaker perturbations that may be important at mesospheric heights. Also shown for reference are lines of constant phase speed of $5,10,20,40$, and $60 \mathrm{~m} \mathrm{~s}^{-1}$. 


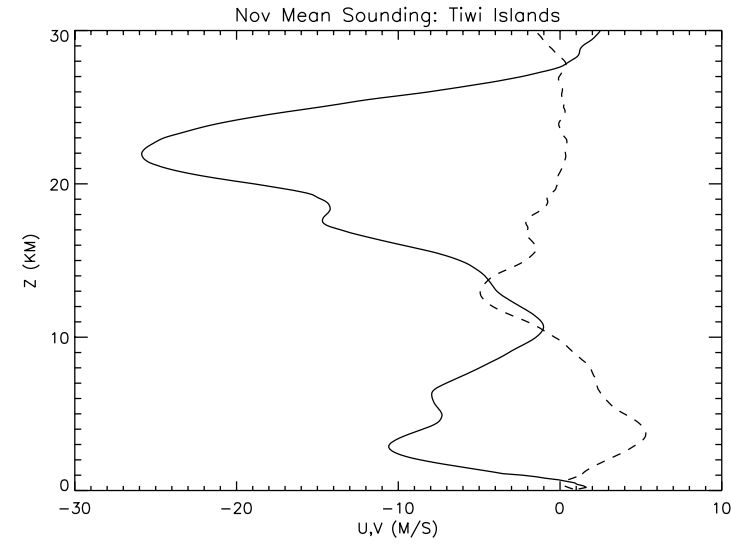

Figure 12. Unmodified mean zonal and meridional wind profiles for the period 15-20 November as measured by 3-hourly radiosondes at Garden Point. These profiles show the stratospheric winds that will subsequently modify the spectra shown in Figures 10 and 11.

upper levels, $\sim 40-50 \mathrm{~m} \mathrm{~s}^{-1}$ based on observations [e.g., Simpson et al., 1993] and model studies of Hector [e.g., Saito et al., 2001; Crook, 2001]. Figure 9a shows a pronounced asymmetry between eastward and westward propagating waves above the largest updraft. Figure $9 \mathrm{~b}$ shows that there is also some north-south asymmetry in the wave amplitudes, but this meridional asymmetry is only rather weak compared to the zonal asymmetry. The zonal asymmetry is mainly due to the wind shear in the upper troposphere (Figure 7), but also partly associated with the lower to middle atmosphere wind effects on wave generation [Beres et al., 2002].

\subsection{Spectral Analysis}

[26] We perform a 3-D space-time cospectral analysis of horizontal and vertical velocity fields to yield cospectra of $u^{\prime}$ and $w^{\prime}$ versus $(k, l, \omega)$ and cospectra of $v^{\prime}$ and $w^{\prime}$ versus $(k, l, \omega)$, where $\left(u^{\prime}, v^{\prime}, w^{\prime}\right)$ is the 3-D wind perturbation relative to the mean flow shown in Figure 7, $\omega$ is the ground-based frequency, and $(k, l)$ is the horizontal wave number. To compute the cospectrum $u^{\prime}$ and $w^{\prime}$ we first compute the 3-D fast Fourier transforms of $u^{\prime}(x, y, t)$ and $w^{\prime}(x, y, t)$ producing the complex transforms $U(k, l, \omega)$ and $W(k, l, \omega)$, respectively. The cospectrum is then given by $\operatorname{Co}(U W)=$ $\operatorname{Re}\left(U \cdot W^{*}\right)$, where $W^{*}$ is the complex conjugate of $W$. We then rebin the cospectra to produce cospectra of $(\phi, c)$ and $(\phi, \kappa)$ where $\phi$ is the wave propagation angle relative to east, $c$ is the phase speed, and $\kappa$ is the horizontal wave number in the direction of propagation. The magnitude of the momentum flux is proportional to $\left[C o(U W)^{2}+C o(V W)^{2}\right]^{1 / 2}$. These spectra are computed at $21-24.75 \mathrm{~km}$ altitude in the stratosphere, then multiplied by the background density $\bar{\rho}$ and averaged in height. ( $\bar{\rho}$ varies from $.08-.04 \mathrm{~kg} \mathrm{~m}^{-3}$ in this height range.) The resulting momentum flux spectra are shown in Figure 10 as polar color contour plots.

[27] Figures 10a-10b show the results for the first 4 hours of the simulation dominated by the Hector storm, and Figures $10 \mathrm{c}-10 \mathrm{~d}$ show the results for the second 4 hours dominated by the squall line convection. The fluxes have been normalized so that red regions have values larger than $97 \%$ of the maximum and white regions have values less than $0.3 \%$ of the maximum fluxes. We give estimates of the net momentum fluxes in section 4 where the model's scale factor is estimated.

[28] Figure 10 shows the dramatic asymmetry favoring eastward relative to westward propagating waves. The eastward flux is 8 times larger than the westward flux in Figures $10 \mathrm{a}-10 \mathrm{~b}$ and almost 5 times larger in Figures 10c$10 \mathrm{~d}$. The largest fluxes are associated with waves in the $c=$ $5-20 \mathrm{~m} \mathrm{~s}^{-1}$ range. In Figures 10a and $10 \mathrm{c} 93 \%$ of the flux is carried by waves with $c<40 \mathrm{~m} \mathrm{~s}^{-1}$. By far, most of the flux is associated with horizontal wavelengths less than $100 \mathrm{~km}$. In Figure 10b, wavelengths $4-50 \mathrm{~km}$ dominate with $74 \%$ of the flux while in Figure 10d more similar fluxes occur for both $4-50 \mathrm{~km}(55 \%)$ and $50-100 \mathrm{~km}$ (38\%) wavelengths. Wavelengths longer than $100 \mathrm{~km}$ represent only $5 \%$ of the flux in Figure $10 \mathrm{~b}$ and $7 \%$ in Figure 10d. These longer wavelengths show a preference for northeastward propagation.

[29] The storm centers move toward the northwest at approximately 5 to $10 \mathrm{~m} \mathrm{~s}^{-1}$ relative to the ground, while the upper troposphere winds are west-southwest at $15 \mathrm{~m} \mathrm{~s}^{-1}$. The mean wind relative to the obstacles is therefore fairly weak, $5-10 \mathrm{~m} \mathrm{~s}^{-1}$ southwestward. The obstacle effect is therefore not expected to be a strong wave generation mechanism [Beres et al., 2002], but any waves generated by this mechanism would appear with northwestward propagation direction relative to the ground and slow phase speeds of less than $10 \mathrm{~m} \mathrm{~s}^{-1}$. Although waves with these characteristics do appear in Figure 10, the fluxes are small. Most of the flux is carried by waves generated via the time variations in the heating field. Note that the $2-\mathrm{km}$ horizontal resolution of the model allows the generation and propagation of waves $20 \mathrm{~km}$ and longer to be simulated without significant numerical dissipation. The peaks in the spectrum occur at wavelengths $40 \mathrm{~km}$ and longer, so these should be well represented in the model.

[30] Figure 10 also shows an asymmetry in the northward and southward fluxes, but it is less pronounced than the

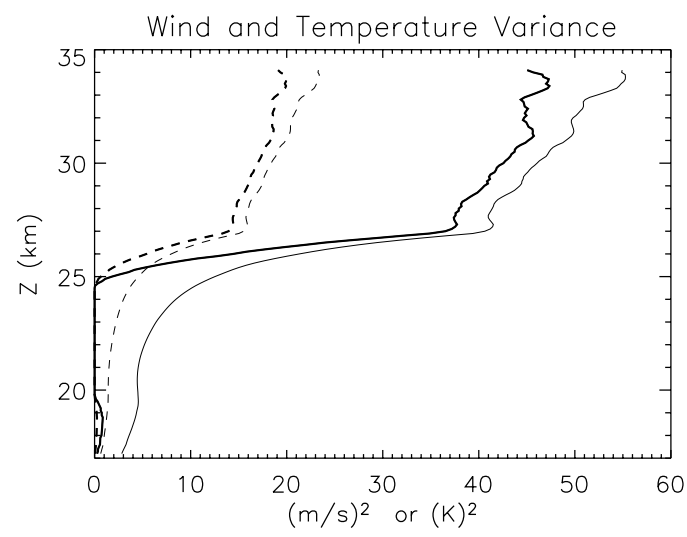

Figure 13. Vertical profiles of horizontal wind variance (solid) and temperature variance (dashed) produced by the ray model with input based on the spectra in Figure 10 as described in the text. The thick curves have been filtered to include only wavelengths $0.2-3.0 \mathrm{~km}$ to match the radiosonde observational analysis. The thin lines include all the waves input. Some features of the observational analysis (Tsuda et al., submitted manuscript, 2004) are reproduced by the model (see text for further details). 
east-west asymmetry. The peak northward fluxes are $\sim 2.5$ times larger than the southward fluxes. Northward waves also show a preference for shorter horizontal wavelengths than southward waves in Figure 10b, while in Figures 10c-10d, northward waves show longer horizontal wavelengths and higher phase speeds than southward waves.

[31] Figure 11 shows results of the same spectral analysis as in Figure 10 but with color contours on a log scale to better show weak perturbations in the stratosphere that may be important at mesopause altitudes where airglow imagers at Katherine and Wyndham observed gravity waves during DAWEX. Although SW propagating waves have weaker amplitudes, $\sim 10^{2}$ smaller than the peak NW propagating waves, they are generated by the convection in the Darwin area and they have high phase speeds, mostly larger than $20 \mathrm{~m} \mathrm{~s}^{-1}$. Slower phase speeds are likely to be filtered by the upper troposphere westward winds. These high phase speed waves are much like those observed by airglow imagers located at Wyndham and Katherine to the SW and SE during DAWEX (P. D. Pautet et al., Climatology of short-period gravity waves observed over northern Australia during the DAWEX campaign and their dominant source regions, submitted to Journal of Geophysical Research, 2004). Note the pronounced lines of enhanced power that curve relative to the lines of constant phase speed. These represent lines of constant vertical wavelength, which deviate from constant phase speed lines for nonhydrostatic waves. The two deepest modes follow lines with $\sim 8$ and $16 \mathrm{~km}$ vertical wavelengths.

[32] The observed background wind profiles derived from the radiosonde observations, extending up to $30 \mathrm{~km}$, are shown in Figure 12. These are identical to those input to the model below $17 \mathrm{~km}$, but differ above. Figure 12 shows the QBO shear at the location of Garden Point at $11.4^{\circ} \mathrm{S}$ latitude. The zonal winds sweep from $-15 \mathrm{~m} \mathrm{~s}^{-1}$ at $17 \mathrm{~km}$ to $-25 \mathrm{~m} \mathrm{~s}^{-1}$ at $22 \mathrm{~km}$, then back to $+2 \mathrm{~m} \mathrm{~s}^{-1}$ at $30 \mathrm{~km}$. Although waves near the peaks in the zonal flux spectrum would not reach critical levels in this QBO wind shear, these waves would still likely interact to some degree with the winds, and could participate in driving mean zonal wind accelerations in the stratosphere. The meridional shear is very weak in the stratosphere. Meridionally propagating waves generated in the model might not interact strongly with the background flow until mesospheric altitudes where the meridional wind fluctuations due to the diurnal tides become substantial.

\section{Model Comparison to DAWEX Observations and Other Studies}

[33] Both the magnitude of the heating input and the magnitude of the gravity wave amplitudes in our model are uncertain and should be considered scalable. This section compares our model results to DAWEX observations and other previous observations and models to assess an appropriate scale factor for our model.

\subsection{Radiosonde Observations}

[34] The radiosondes were able to observe gravity waves with vertical wavelengths as large as $15 \mathrm{~km}$, but the analysis techniques applied to analyze these profiles for waves tend to emphasize short vertical wavelength waves $\sim 2-3 \mathrm{~km}$ (Tsuda et al., submitted manuscript, 2004). A 3-km vertical wavelength wave has an intrinsic phase speed of $\sim 13 \mathrm{~m} \mathrm{~s}^{-1}$. This is smaller than the intrinsic phase speeds of many of the waves in the spectra in Figures 10 and 11. Considering the background wind profile in Figure 12 and the peak in the momentum flux spectrum at $2-10 \mathrm{~m} \mathrm{~s}^{-1}$ corresponding to waves propagating to the northeast, the peak intrinsic phase speeds will be $\sim 12-30 \mathrm{~m} \mathrm{~s}^{-1}$ in the lower stratosphere, corresponing to vertical wavelengths $\sim 2.8-7 \mathrm{~km}$.

[35] One feature of the waves with vertical wavelengths $<3 \mathrm{~km}$ derived from the radiosonde analysis (Tsuda et al., submitted manuscript, 2004) is a double-peaked variance profile in the stratosphere temperature and horizontal wind variances: A lower peak at $\sim 18 \mathrm{~km}$ decays to very weak variances between 20 and $25 \mathrm{~km}$, then a second peak emerges above $25 \mathrm{~km}$. To investigate the relevance of our model-derived wave spectrum to the shape of this profile, we run a ray-tracing model [Alexander, 1998] using the observed background wind and temperature profiles. The ray model requires an input spectrum of waves. We choose a single northeastward propagation direction and horizontal wavelength of $40 \mathrm{~km}$, and a spectrum of phase speeds peaking at $4 \mathrm{~m} \mathrm{~s}^{-1}$ which mimics the northeastward momentum flux spectral peaks in Figure 10. The flux was set to match $2.5 \times$ local values in the model or $50 \mathrm{mPa}$. The spectrum of waves propagating through the observed winds experience Doppler shifting and refraction, which changes the waves' vertical wavelengths with height.

[36] Figure 13 shows profiles of the horizontal wind and temperature variance from this ray model. The thick lines show the variance for vertical wavelengths $0.2-3 \mathrm{~km}$ only, while the thin lines show the variance from all the waves input into the ray model. This experiment suggests that the decrease in variance between 20 and $25 \mathrm{~km}$ in the observations may be at least in part caused by the vertical wavelength filtering in the data analysis method. It also suggests the rapid increase in variance above $25 \mathrm{~km}$ in the observations could be caused by the type of waves generated in our model, and this result helps to validate our momentum flux spectra. This result is rather insensitive to the wave amplitudes and horizontal wave numbers, but is sensitive to their phase speeds and propagation directions. It is difficult to make the comparison between the observations and the model quantitatively without further work because the averaging timescales in both the data and model and the areal averaging in the model (required to compute the spectrum) lead to large uncertainties in the magnitudes in the variance calculations. The lower altitude peak in the observed variance below $20 \mathrm{~km}$ is much larger than that produced by the ray model. This implies this lower altitude peak is probably largely due to larger-scale waves that are not resolved in our model study, and which may be generated by sources outside of the local Darwin area.

\subsection{Garden Point Wind Profiler Data}

[37] The tropospheric perturbations in the model at the model grid point closest to Garden Point can be compared to the radar wind profile that was located there during DAWEX. The perturbations in the model and the data for the time period 0530-1100 UT are shown in the companion 
paper [Vincent et al., 2004]. The observations were filtered to include fluctuations with periods $24 \mathrm{~min}$ to 3 hours, and the model for fluctuations shorter than 3 hours. There are some remarkably similar features in both the model and the data. However, the wave amplitudes in the model are roughly 5-10 times smaller than the observations. This difference at face value would suggest that the model output wave amplitudes should be scaled upward by about a factor of $5-10$.

\subsection{Peak Updraft Velocities}

[38] Peak updraft vertical velocities in the model are $\sim 11 \mathrm{~m} \mathrm{~s}^{-1}$. In contrast, observations of convection in this area from earlier campaigns suggest peak updraft velocities of 40-50 $\mathrm{m} \mathrm{s}^{-1}$ for major Hector events [Simpson et al., 1993] such as the 17 November case we are studying. Model studies of Hector convection have reported updrafts of 30-35 m s${ }^{-1}$ [Saito et al., 2001; Crook, 2001; Lane et al., 2001], though these updraft magnitudes are described as moderate Hector events [Crook, 2001]. Model studies such as these might be expected to be a bit weaker than observed due to model resolution and numerical dissipation issues. These studies suggest our input heating and output amplitudes should be scaled upward by a factor of $\sim 3-5$.

\subsection{Stratospheric Wave Amplitudes}

[39] Studies of stratospheric waves generated by tropical convection include both observational and modeling studies. Alexander et al. [2000] derived gravity wave momentum fluxes from 3-D vector winds measured in situ on board the ER-2 aircraft in the lower stratosphere above deep convective clouds. Magnitudes directly above the deepest clouds averaged $130 \mathrm{mPa}$. Radar observations of stratospheric waves above a Typhoon over Japan showed momentum flux magnitudes of $\sim 30-100 \mathrm{mPa}$ above the most active convection [Sato, 1993]. Local measures of momentum fluxes in the model stratosphere above the strongest heating centers averaged over a $40 \times 40 \mathrm{~km}$ area are $\sim 20 \mathrm{mPa}$. These comparisons imply a scaling factor in the range $\sqrt{1.5}-\sqrt{6.5}$ should be applied to our model. The observational range is wide, and it is difficult to judge precisely how to compare the model results to these observations. The averaging time and area will both strongly influence the result.

[40] Model studies of gravity waves generated by convection show very similar patterns of gravity wave perturbations as in our Figure 8 [Lane et al., 2001; Horinouchi et al., 2002]. The study by Lane et al. [2001] gives peak wave vertical velocity amplitudes of $\sim 0.5 \mathrm{~m} \mathrm{~s}^{-1}$ in the stratosphere above the active convective centers. Peak vertical velocities of stratospheric waves in our model before scaling are $\sim 0.3 \mathrm{~m} \mathrm{~s}^{-1}$. However, the tropospheric vertical velocities in the Lane et al. [2001] study, as described in section 4.3, are more characteristic of moderate Hector events, while our study includes a major Hector event. So the wave amplitudes in the work of Lane et al. [2001] should be somewhat lower than for our case, consistent with a scale factor $\geq \sqrt{3}$. Horinouchi et al. [2002] studied gravity waves generated by tropical convection and propagation into the mesosphere. They report rain rates, but not tropospheric vertical velocities, and the convection they model is oceanic rather than the stronger island and conti- nental convection we study here. Vertical velocities at $30 \mathrm{~km}$ in their model are $\sim 0.5 \mathrm{~m} \mathrm{~s}^{-1}$, and momentum fluxes averaged over their $200 \times 200-\mathrm{km}$ domain and over 24 hours are $\sim 0.01 \mathrm{mPa}$ at $20 \mathrm{~km}$. These would equate to some much larger value for local regions within the domain averaged over shorter time periods, but we cannot compare directly with these results.

\section{Conclusions}

[41] We have described a model study of gravity waves generated by convection in the Darwin area on 17 November 2001. The waves are forced by a very realistic spatially and temporally varying latent heating field derived from precipitation radar measurements. The resulting wave field has many very realistic properties that compare well to observations, but the magnitudes are highly uncertain. The model resolution $(2 \mathrm{~km})$ and domain size allowed us to study convectively generated waves with horizontal wavelengths $\sim 20-400 \mathrm{~km}$. We ran the model for 8 hours and saved the output every $3 \mathrm{~min}$, so we could resolve waves with frequencies between 6 min and 8 hours.

[42] We also examined the morphology and time variations in our derived latent heating field. The first half of the period we studied was dominated by a major Hector event over the Tiwi Islands. The second half included a continental squall line to the southeast of Darwin, and there is a minimum in convective activity between these two events. The heating centers have small scales, commonly $\sim 8-16 \mathrm{~km}$, with slightly longer scales occurring in the second half of the period.

[43] Spectral analysis showed a pronounced preference for eastward propagating waves over their westward propagating counterparts. This asymmetry in the wave field is cause by the upper troposphere wind shear, with a secondary effect of lower and middle tropospheric winds in the region of the wave forcing. There is also a preference for northward over southward propagating waves in the stratosphere, but this asymmetry is less pronounced. The wave phase speeds range from 0 to $80 \mathrm{~m} \mathrm{~s}^{-1}$ with a distinct peak at low northeastward phase speeds $5-20 \mathrm{~m} \mathrm{~s}^{-1}$. Significant fluxes occur out to phase speeds of $40 \mathrm{~m} \mathrm{~s}^{-1}$, with higher phase speeds carrying $\leq 7 \%$ of the total flux. Peak fluxes occur for waves with horizontal wavelengths of $20-100 \mathrm{~km}$, while longer wavelength waves account for $\leq 7 \%$ of the flux.

[44] We calibrate the model against several observational standards, and the comparison suggests our model wave amplitudes are roughly a factor $2-10 \times$ too small. The high end of this range, $5-10 \times$, comes from a comparison to tropospheric waves in VHF radar observations [Vincent et al., 2004]. It is possible that there are fewer trapped tropospheric waves in our model relative to observations for two reasons: (1) The resolution of our simulation only permits waves with horizontal wavelengths $>20 \mathrm{~km}$ to propagate without significant numerical dissipation, and shorter waves would be most commonly trapped. (2) Convection commonly generates a layer of low stability in the upper troposphere which can effectively trap frequencies higher than the minimum in the buoyancy frequency profile [Pandya and Alexander, 1999], but our simulation includes a background profile without this pronounced low stability 
layer. If wave trapping is greater in the observations than in the model, this could explain some fraction of the differences in the wave amplitudes.

[45] Other scale factor indicators place the most likely scale factor in the range of $2-5 \times$. This range suggests the momentum fluxes entering the stratosphere averaged over the $400 \times 400 \mathrm{~km}$ model domain and over each 4-hour period, and integrated over the spectrum are roughly $\sim 1-$ $2 \mathrm{mPa}$ for the first period and $\sim 2-5 \mathrm{mPa}$ for the second. Note that because the waves in our study are generated by localized sources with high temporal variability, the magnitude of this flux is very sensitive to the spatial and temporal averaging. This will likely be true in general for waves generated by convection, so these effects should be considered carefully before comparing gravity wave momentum fluxes from different types of satellite, aircraft, and ground-based observations [e.g., Alexander and Holton, 2004; M. Ern et al., Absolute values of gravity wave momentum flux derived from satellite data, submitted to Journal of Geophysical Research, 2004].

[46] $\sim 40 \%$ of the flux propagates to the northeast with $0-10 \mathrm{~m} \mathrm{~s}^{-1}$ ground-relative phase speeds, and another $\sim 20 \%$ propagates northeastward with $10-40 \mathrm{~m} \mathrm{~s}^{-1}$ phase speeds. This preference for northeastward wave propagation tells us in hindsight that the DAWEX airglow imagers to the southeast and southwest of Darwin were not ideally placed for observing the largest amplitude waves generated by convection in the Darwin area.

[47] Some features of waves observed in radar wind profiler and radiosonde measurements are reproduced by the model. Further work is warranted to make more detailed and quantitative comparisons between our model and DAWEX observations.

[48] Acknowledgments. This work was supported by the National Science Foundation Physical Meteorology Program, NSF grant ATM0234230, and the NASA Atmospheric Chemistry Modeling and Analysis Program grant NASW-01017. The authors also thank Jim Hecht, Andrew MacKinnon, Gary Swenson, Mike Taylor, Bob Vincent, and Matthew Wheeler for valuable comments on the manuscript.

\section{References}

Alexander, M. (1998), Interpretations of observed climatological patterns in stratospheric gravity wave variance, J. Geophys. Res., 103, 86278640 .

Alexander, M., and J. Holton (2004), Gravity waves generated by a transient localized heat source, Atmos. Chem. Phys., 4, 923-932.

Alexander, M., J. Holton, and D. Durran (1995), The gravity wave response above deep convection in a squall line simulation, J. Atmos. Sci., 52, $2212-2226$.

Alexander, M., J. Beres, and L. Pfister (2000), Tropical stratospheric gravity wave activity and relationship to clouds, J. Geophys. Res., 105(D17), 22,299-22,309

Beres, J., M. Alexander, and J. R. Holton (2002), Effects of tropospheric wind shear on the spectrum of convectively generated gravity waves, J. Atmos. Sci., 59, 1805-1824.

Beres, J., M. J. Alexander, and J. R. Holton (2004), A method of specifying the gravity wave spectrum above convection based on latent heating properties and background wind, J. Atmos. Sci., 61, 324-337.

Bergman, J. W., and M. L. Salby (1994), Equatorial wave activity derived from fluctuations in observed convection, J. Atmos. Sci., 51, 3791-3806.

Chun, H.-Y., and J.-J. Baik (2002), An updated parameterization of convectively forced gravity wave drag for use in large-scale models, J. Atmos. Sci., 59, 1006-1017.

Crook, N. A. (2001), Understanding Hector: The dynamics of island thunderstorms, Mon. Weather Rev., 129(6), 1550-1563.
Durran, D., and J. B. Klemp (1983), A compressible model for the simulation of moist mountain waves, Mon. Weather Rev., 111, 2341-2361.

Durran, D., M.-J. Yang, D. Slinn, and R. Brown (1993), Toward more accurate wave-permeable boundary conditions, Mon. Weather Rev., 121, 604-620.

Garcia, R., and M. Salby (1987), Transient response to localized episodic heating in the tropics. Part ii: Far-field behavior, J. Atmos. Sci., 44, 499530 .

Holton, J. (1972), Waves in the equatorial stratosphere generated by tropospheric heat sources, J. Atmos. Sci., 29, 368-375.

Holton, J., J. Beres, and X. Zhou (2002), On the vertical scale of gravity waves excited by localized thermal forcing, J. Atmos. Sci., 59, 20192023.

Horinouchi, T., T. Nakamura, and J.-I. Kosaka (2002), Convectively generated mesoscale gravity waves simulated throughout the middle atmosphere, Geophys. Res. Lett., 29(21), 2007, doi:10.1029/2002GL016069.

Keenan, T., K. Glasson, T. Cummings, J. Keeler, and J. Lutz (1998), The bmrc/ncar c-band polarimetric (c-pol) radar system, J. Atmos. Ocean Technol., 15, 871-886.

Lane, T., and M. Reeder (2001), Modelling the generation of gravity waves by a maritime continent thunderstorm, Q. J. R. Meteorol. Soc., 127, $2705-2724$

Lane, T., M. Reeder, and T. Clark (2001), Numerical modeling of gravity wave generation by deep tropical convection, J. Atmos. Sci., 58(10), $1249-1274$

Manzini, E., and K. Hamilton (1993), Middle atmospheric traveling waves forced by latent and convective heating, J. Atmos. Sci., 50, 2180-2200.

May, P., A. Jameson, T. Keenan, P. Johnston, and C. Lucas (2002), Combined wind profiler/polarimetric radar studies of the vertical motion and microphysical characteristics of tropical sea-breeze thunderstorms, Mon. Weather Rev., 130(9), 2228-2239.

Pandya, R., and M. Alexander (1999), Linear stratospheric gravity waves above convective thermal forcing, J. Atmos. Sci., 56(14), 2434-2446.

Pandya, R., D. Durran, and C. Bretherton (1993), Comments on "Thermally forced gravity waves in an atmosphere at rest," J. Atmos. Sci., 50, 4097-4101.

Piani, C., and D. Durran (2001), A numerical study of stratospheric gravity waves triggered by squall-lines observed during the TOGA-COARE and COPT-81 experiments, J. Atmos. Sci., 58, 3702-3723.

Piani, C., D. Durran, M. Alexander, and J. Holton (2000), A numerical study of three-dimensional gravity waves triggered by deep tropical convection and their role in the dynamics of the QBO, J. Atmos. Sci., 57(22), $3689-3702$.

Ricciardulli, L., and R. Garcia (2000), The excitation of equatorial waves by deep convection in the NCAR Community Climate Model (CCM3), J. Atmos. Sci., 57(21), 3461-3487.

Saito, K., T. Keenan, G. Holland, and K. Puri (2001), Numerical simulation of the diurnal evolution of tropical island convection over the Maritime Continent, Mon. Weather Rev., 129(3), 378-400.

Salby, M. L., and R. Garcia (1987), Transient response to localized episodic heating in the tropics. Part I: Excitation and short-time near-field behaviour, J. Atmos. Sci., 44, 458-498.

Sato, K. (1993), Small-scale wind disturbances observed by the mu radar during the passage of typhoon kelly, J. Atmos. Sci., 50, 518-537.

Simpson, J., T. Keenan, B. Ferrier, R. Simpson, and G. Holland (1993), Cumulus mergers in the Maritime Continent region, Meteorol. Atmos. Phys., 51, 73-99.

Song, I.-S., H.-Y. Chun, and T. P. Lane (2003), Generation mechanisms of convectively forced internal gravity waves and their propagation to the stratosphere, J. Atmos. Sci., 60, 1960-1980.

Steiner, M., J. Smith, and R. Uijlenjoet (2004), A microphysical interpretation of radar reflectivity-rain rate relationships, J. Atmos. Sci., 61, 1114-1131.

Vincent, R. A., A. MacKinnon, I. M. Reid, and M. J. Alexander (2004), VHF profiler observations of winds and waves in the troposphere during the Darwin Area Wave Experiment (DAWEX), J. Geophys. Res., D20S02, doi:10.1029/2004JD004714.

Xue, M., D. Wang, J. Gao, K. Brewster, and K. Droegemeier (2003), The Advanced Regional Prediction System (ARPS), storm-scale numerical weather prediction and data assimilation, Meteorol. Atmos. Phys., 82, doi:10.1007/s0,703-001-0595-6, 139-170.

M. J. Alexander, Colorado Research Associates, Division of Northwest Research Associates, 3380 Mitchell Lane, Boulder, CO 80301, USA. (alexand@colorado-research.com)

J. H. Beres, NCAR, Boulder, CO 80305, USA

P. T. May, BMRC, GPO Box 1289K, Melbourne, VIC 3001, Australia. 